\title{
Factors affecting elemental quantification at the atomic scale using EELS
}

\author{
R. Srinivasan, G.B. Viswanathan, J. Sosa, D.W. McComb”, J. Ringnalda*, H.L. Fraser
}

Center for the Accelerated Maturation of Materials and Department of Materials Science and Engineering, The Ohio State University, Columbus, OH 43210, U.S.A.

\#Department of Materials, Imperial College London, London, SW7 2AZ, United Kingdom

*FEI Company, 5350 NE Dawson Creek Drive, Hillsboro, OR 97124, USA

Advances in aberration corrected high resolution scanning transmission electron microscopy (HRSTEM) [1,2], along with rapid advances in instrumentation, have made chemical analysis at the atomic scale a reality. Several recent studies have demonstrated the ability for qualitative and semi-quantitative determination of elemental concentrations at the atomic scale, using electron energy-loss (EELS) spectroscopy [3-5]. However, the influence of key factors dependent upon the specimen as well as the imaging conditions still merits further study. The current study focuses on understanding the effect of some of these parameters through EELS analysis on strontium titanate, using a FEI Titan 80-300 S/TEM operated at $300 \mathrm{kV}$, equipped with a monochromator, CEOS third-order probe aberration corrector and Gatan Tridiem 866 imaging filter.

Figure 1a shows the structure of the $\mathrm{SrTiO}_{3}$ unit cell, while Figure 1b shows a HAADF STEM image of $\mathrm{SrTiO}_{3}$ (bright atoms - Sr, darker atoms - Ti) along the [001] zone. Figure 2 shows the results of spatially resolved EELS experiments performed to determine elemental concentrations in the $\mathrm{SrTiO}_{3}$ lattice. The individual elemental edges can be distinctly detected at all probe positions, suggesting a common de-channeling effect through the sample thickness. This is of interest since in previous work [5] it was found that the EELS signal is confined to given atomic columns. Figure 2a shows an EELS profile along alternating columns of $\mathrm{Sr}$ and $\mathrm{Ti} / \mathrm{O}$ where compositional variations are as expected and in concert with the conclusion [5]. However, inspection of the EELS profiles in figures $2 \mathrm{~b}$ and $\mathrm{c}$ reveals a different behavior. Thus, in figure $2 \mathrm{~b}$, profiling across alternating columns of $\mathrm{Sr}$ and $\mathrm{O}$, it is observed that where $\mathrm{O}$ peaks, so does $\mathrm{Ti}$, although the Ti columns lie in rows adjacent to the profile. Similarly, in figure $2 \mathrm{c}$, the $\mathrm{O}$ signal should be flat, but actually peaks at $\mathrm{Ti} / \mathrm{O}$ columns. These preliminary results from Figure $2 \mathrm{~b}$ and $2 \mathrm{c}$ imply a significant delocalization, or column jumping, of the signal when the electrons are scattered by the $\mathrm{O}$ (and other atomic) columns. Direct interpretation of the EELS signal is thus complicated, in accordance with the warning in [5]. More detailed studies, aimed at exploring the effect of specimen thickness, convergence angle and beam energy spread, supplemented with results of image simulations, will be presented.

\section{References}

1. M. Haider, H. Rose et al., Ultramicroscopy 75, 53 (1998).

2. M. Haider, S.Uhlemann et al., Nature 392, 768 (1998).

3. D.A. Muller, Y. Tzou, R. Raj, J. Silcox, Nature 366, 725 (1993).

4. E.C. Cosgriff, M.P. Oxley, L.J. Allen, S.J. Pennycook, Ultramicroscopy 102, 317 (2005).

5. L.J. Allen, S.D. Findlay, A.R. Lupini, M.P. Oxley, S.J. Pennycook, Phys. Rev. Lett. 91, 105503-1 (2003). 


\section{Figures}

(a)

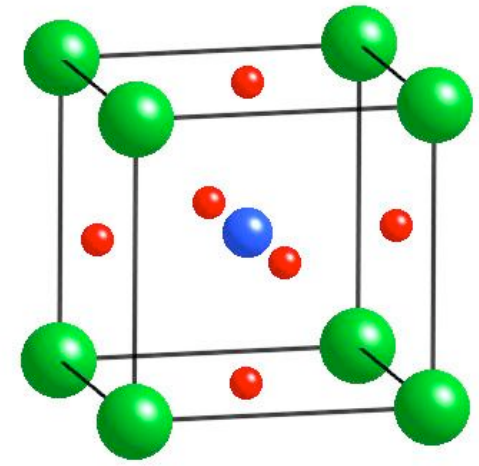

$\mathrm{OSr}$

$\mathrm{OTi}$

00 (b)

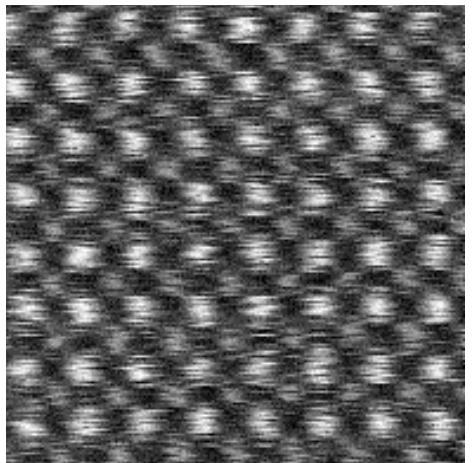

Figure 1: (a) Schematic of $\mathrm{SrTiO}_{3}$ unit cell, (b) HAADF-HRSTEM image along [001] zone showing brighter $\mathrm{Sr}$ and darker $\mathrm{Ti} / \mathrm{O}$ columns. Pure $\mathrm{O}$ columns are not visible in this image.
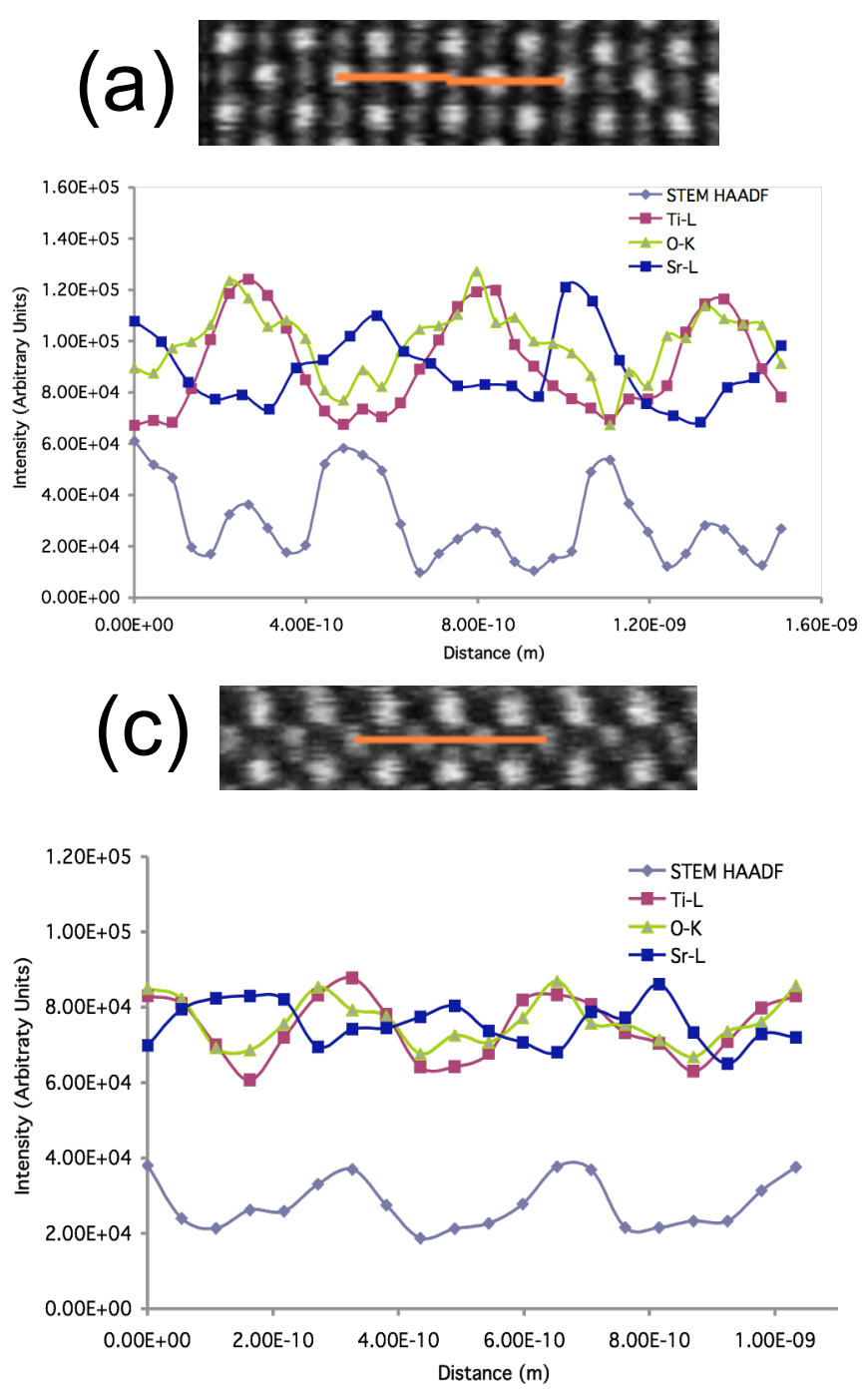

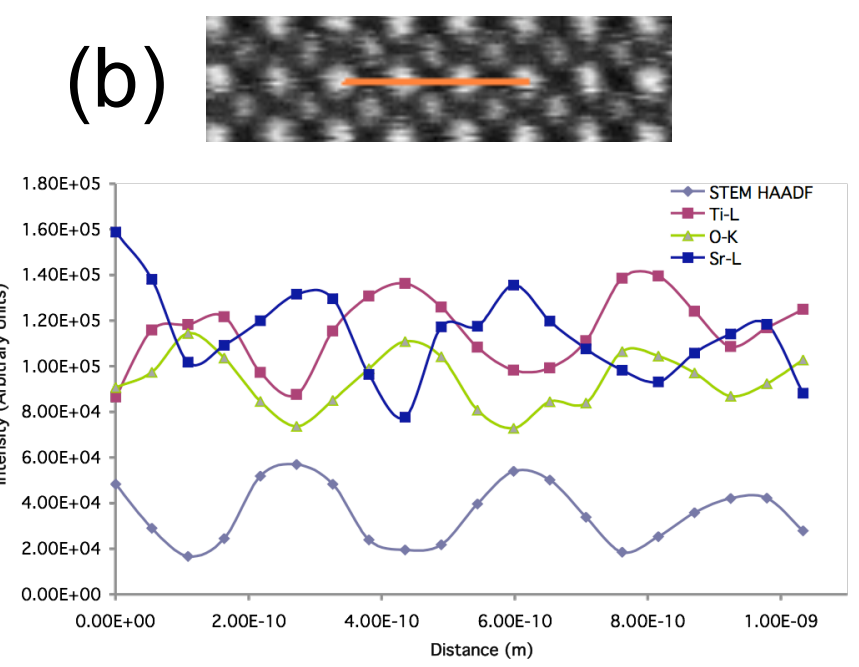

Figure 2: EELS signals for various line profiles across (a) Sr-Ti/O-Sr columns, (b) Sr-O-Sr columns, (c) Ti/O-O-Ti/O columns. Corresponding HAADFHRSTEM images for each case are also shown 